\title{
COUNTABLE PERIODIC $C$-GROUPS AS AUTOMORPHISM GROUPS
}

\author{
by MARTYN R. DIXON
}

(Received 30th July 1990)

It is shown that if $G$ is a group and Aut $G$ is a countable periodic $C C$-group then Aut $G$ is $F C$.

1980 Mathematics subject classification (1985 Revision). 20F28.

\section{Introduction}

The thrust of several recent papers (see, for example, [2, 7, 9 and 11]) has been to show that certain kinds of infinite group cannot occur as the full automorphism group, Aut $G$, of some group $G$. Indeed in [2, Theorem A], [7, Theorem] and [9, Theorem A] it is shown that if Aut $G$ is periodic and satisfies some other appropriate hypothesis then the divisible radical of Aut $G$ is trivial. (Here, the divisible radical of a group is the unique largest radicable abelian subgroup, if it exists.) In this short note we add one further result of this type and hence provide further examples of groups which cannot be automorphism groups. For example a countable direct product of Cernikov groups, at least one of which is infinite, can never be an automorphism group.

In this paper we shall be concerned with the class of $C C$-groups. A group $G$ is called a $C C$-group if $G / C_{G}\left(x^{G}\right)$ is Cernikov for all $x \in G$; if $G / C_{G}\left(x^{G}\right)$ is finite for all $x \in G$ then we call $G$ an $F C$-group. The class of $C C$-groups has been the subject of much recent work (see, for example, [1, 4 and 6].) Furthermore, Zimmerman [11] has considered countable, periodic $F C$-groups as automorphism groups and showed there that with certain extra hypotheses the structure of Aut $G$ is very restricted. Our main result is as follows.

Theorem. Suppose $G$ is a group such that Aut $G$ is a countable periodic CC-group. Then Aut $G$ is an FC-group.

The main part of the argument here is to show using the techniques of Lemma 2 of [11] that $G$ must be a $C C$-group. We remark in passing that it seems reasonable to expect that the theorem holds in the absence of countability; however, this extra hypothesis is necessary to show that $G$ is $C C$. In [5], Menegazzo and Stonehewer have 
given an example of a countable group $G$ which is not $C C$ but which has uncountable, elementary abelian automorphism group.

Our notation is standard and is that used in [10].

\section{The proof of the theorem}

Our first result is well known but we include it for completeness.

Lemma 1. Suppose $G$ is a $C C$-group. Then $G$ has a periodic divisible radical $D$ and $G / D$ is an $F C$-group.

Proof. It follows using arguments similar to those of Lemma 3 of [1] that the semiradicable radical, $R$, of $G$ is nilpotent of class 2. Furthermore if $M, L$ are periodic divisible subgroups of $G$ then $M, L \leqq R$ so are subnormal in $G$. Hence by [10, vol. I, Lemma 4.46], $G$ has a periodic divisible radical $D$, lying in $R$ and $D$ is the torsion subgroup of $R$, by [10, vol. II, Theorem 9.23]. If bars denote groups modulo $R$ and if $x \in G$ then $x^{G}$ is Cernikov-by-cyclic (see [10, vol. I, Theorem 4.36]). Hence $\bar{x}^{\bar{G}} \cong x^{G} / x^{G} \cap$ $R$ is finite-by-cyclic so $\bar{G}$ is $F C$ by [10, vol. I, Corollary 3, p. 122]. Also if $T$ is the torsion subgroup of $G$ then [10, vol. I, Corollary, p. 129] implies $G / T$ is abelian. Hence $G /(T \cap R)=G / D$ is an $F C$-group as required.

In their recent paper [4], Franciosi, de Giovanni and Tomkinson remark that if $G$ is a $C C$-group and $M$ is a normal subgroup with $G / M$ Cernikov then one cannot necessarily find a normal Cernikov subgroup $K$ such that $G=M K$. Our next results are aimed at showing that this can be done in certain cases, which are of importance to our situation.

Lemma 2. Suppose $D$ is a periodic $C C$-group and $M$ is an abelian normal subgroup such that $D / M$ is a divisible abelian group. Then $D$ is abelian.

Proof. Suppose $x \in M$. Then $x^{D}$ is Cernikov by [10, vol. I, Theorem 4.36(ii)] and abelian. It follows from [10, vol. I, Corollary, p. 85] that $D / C_{D}\left(x^{D}\right)$ is finite and also divisible. Hence $M \leqq Z(D)$, and $D$ is nilpotent of class at most 2. Furthermore $D / Z(D)$ is divisible so the same argument implies $D$ is abelian, as required.

Lemma 3. Let $G$ be a periodic $C C$-group and suppose $M$ is a normal abelian subgroup of finite exponent such that $G / M$ is Cernikov. Then $G$ has a normal Černikov subgroup $K$ such that $G=M K$.

Proof. Let $D / M$ be the divisible radical of $G / M$. Then $G / D$ is finite so there is a finite subset $X$ of $G$ such that $G=D X^{G}$. Note that $X^{G}$ is Cernikov.

By Lemma $2, D$ is abelian. Let $B$ be a basic subgroup of $D$. Then $B M / M$ is a subgroup of the Cernikov group $D / M$ and hence has finite rank. Hence $B / M \cap B$ is finite and $B$ has finite exponent. Thus $D=B \oplus R$ for some (characteristic) divisible 
group $R$. Now $R$ must be Cernikov since $M$ has finite exponent. Since $B / M \cap B$ is finite, $D$ has a finite subset $Y$ such that $B=Y(M \cap B)$. Hence $G=X^{G} D=X^{G} Y^{G} R M$ and we set $K=X^{G} Y^{G} R$, a Cernikov normal subgroup of $G$.

Our next result uses the argument of Lemma 2 of [11]. We let $Z$ denote the centre of a group $G$.

Lemma 4. Let $G$ be a group and suppose Aut $G$ is a countable periodic CC-group. Then $G$ is a CC-group.

Proof. Let $Q=G / Z$. If $x \in G$ let $R=x^{G} / x^{G} \cap Z, M=C_{G}(R)$ and $L=C_{G}\left(x^{G}\right)$. Since $G / Z$ is a periodic $C C$-group, $R$ is Černikov. By a theorem of Baer [10, vol. I, Theorem 3.29] it follows that $G / M$ is Cernikov. Let $D / M$ be its divisible radical and let bars denote groups modulo $L$. Then $\bar{G}$ is a periodic $C C$-group. There is a monomorphism from $\bar{M}$ to $\operatorname{Hom}\left(x^{G}, x^{G} \cap Z\right)$ defined by $m L \mapsto f_{m}$, for $m \in M$, where $f_{m}(g)=g^{-1} g^{m}$ for $g \in x^{G}$. Since $Z \cap x^{G} \leqq \operatorname{ker} f_{m}$ there is an induced map $\bar{f}_{m}$ from $x^{G} /\left(x^{G} \cap Z\right)\left(x^{G}\right)^{\prime}$ to $x^{G} \cap Z$. Note that $f_{m}$ and $f_{m}$ have the same order. Since $x^{G} /\left(x^{G} \cap Z\right)\left(x^{G}\right)^{\prime}$ is Cernikov it follows that $\operatorname{Hom}\left(x^{G} /\left(x^{G} \cap Z\right)\left(x^{G}\right)^{\prime}, x^{G} \cap Z\right)=K \oplus J$ where $K$ is torsion-free and $J$ is an abelian $\pi$-group of finite exponent, where $\pi$ is the set of primes dividing the orders of the elements of $Z$. Since $\bar{M}$ is periodic it follows that $\bar{M}$ is an abelian $\pi$-group of finite exponent.

Now by Lemma $3, \bar{G}=\bar{N} \bar{M}$ with $\bar{N}$ a Cernikov normal subgroup of $\bar{G}$. Since $\bar{G} / \bar{N}$ is an abelian group of finite exponent and an image of $Q_{a b}$, it follows by [11, Lemma 1] that $\bar{G} / \bar{N}$ is finite. (It is here that we require the countability of Aut $G$ ). Hence $\bar{G}$ is Cernikov and $G$ is a $C C$-group, as required.

Corollary. If $G$ is a group and Aut $G$ is a periodic countable $C C$-group then $G / Z$ is finite.

Proof. By Lemma $1, G$ is a $C C$-group and $G / Z$ is periodic. Since Aut $G$ is countable, Theorem 4.5 of [6] shows that $G / Z$ is a Cernikov group. Let $D / Z$ be the divisible radical of $G / Z$. Since a periodic group of automorphisms of an abelian Cernikov group is finite, it follows that $G / C_{G}(D / Z)$ is finite. A result of Pettet [8, Proposition 5.5] now shows that $G / Z$ has finite exponent. It follows that $G / Z$ is finite.

\section{Proof of the Theorem.}

We shall let $D$ denote the divisible radical of Aut $G$, which exists by Lemma 1 . We show that $D=1$.

Since $D$ is divisible it must act trivially on $G / Z$ which is finite, by the above corollary. Hence

$$
[G, D] \leqq Z
$$


Also if $T$ is the torsion subgroup of $G$ and if $A=C_{\text {Aut } G}(G / Z)$ then Proposition 3.8 of [8] shows that $A / C_{A}(G / T)$ has exponent dividing 12 . Since $D \leqq A$ it follows that

$$
[G, D] \leqq T
$$

Hence $[G, D] \leqq T \cap Z=T(Z)$, the torsion subgroup of $Z$.

On the other hand, since $G / Z$ is finite, $G$ is finite-by-abelian and hence [3, Corollary 2] implies the Sylow $p$-subgroups of $G$ are finite. Hence $D$ acts trivially on $T(Z)$. Finally if $\alpha \in D$ consider the map $\alpha \mapsto[g, \alpha]$, for fixed $g \in G$. Since $[g, \alpha] \in T(Z) \leqq C_{G}(D)$ this map is a homomorphism. Hence $[g, D]$ is a periodic divisible subgroup of $T(Z)$ whence

$$
[g, D]=1 \text {. }
$$

Thus, $D$ acts trivially on $G$ and $D=1$ as required.

We remark finally that the proof shows that if Aut $G$ is a countable periodic $F C$-group then the divisible radical of Aut $G$ is trivial, which can of course be deduced from the work of Zimmerman.

Acknowledgements. The author would like to thank the referee for several suggestions concerning this paper, and also wishes to thank the staff at the Mathematics Instititute, University of Warwick for their hospitality whilst this research was being done.

\section{REFERENCES}

1. J. Alcazar and J. Otal, Sylow subgroups of groups with Cernikov conjugacy classes, J. Algebra 110 (1987), 507-513.

2. M. R. Dixon and M. J. Evans, Periodic divisible-by-finite automorphism groups are finite, $J$. Algebra 137 (1991), 416-424.

3. S. Franciosi and F. de Giovanni, A note on groups with countable automorphism groups, Arch. Math. 47 (1986), 12-16.

4. S. Francosi, F. de Giovanm and M. J. Tomkinson, Groups with Cernikov conjugacy classes, J. Austral. Math. Soc. Ser. A 50 (1991), 1-14.

5. F. Menegazzo and S. E. Stonehewer, On the automorphism group of a nilpotent p-group, $J$. London Math. Soc. (2) 31(1985), 272-276.

6. J. Otal, J. M. Peña and M. J. Tomkinson, Locally inner automorphisms of $C C$-groups, $J$. Algebra 141 (1991), 382-398.

7. M. R. Pettet, Locally finite groups as automorphism groups, Arch. Math. 48 (1987), 1-9.

8. M. R. Pettet, Almost-nilpotent periodic groups as automorphism groups, Quart. J. Math. Oxford (2) 41 (1990), 93-108.

9. D. J. S. Robinson, Infinite torsion groups as automorphism groups, Quart. J. Math. Oxford (2) 30 (1979), 351-364. 
10. D. J. S. Robinson, Finiteness Conditions and Generalized Soluble Groups, vols. I and II (Springer, Berlin-Heidelberg-New York, 1972).

11. J. Zimmerman, Countable torsion $F C$-groups as automorphism groups, Arch. Math. 43 (1984), 108-116.

Mathematics Department

UNIVERSTTY OF ALABAMa

Box 870350

TuscaloOSA, AL 35487-0350

USA 